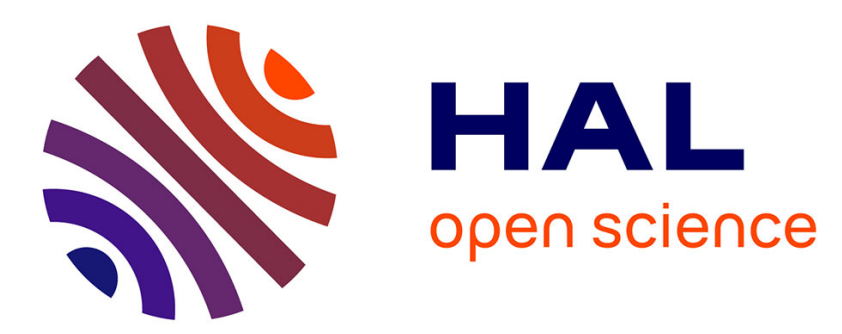

\title{
Acoustical inverse problems regularization: direct definition of filter factors using Signal-to-Noise Ratio
}

Philippe Gauthier, Anthony Gerard, Cedric Camier, Alain Berry

\section{To cite this version:}

Philippe Gauthier, Anthony Gerard, Cedric Camier, Alain Berry. Acoustical inverse problems regularization: direct definition of filter factors using Signal-to-Noise Ratio. Acoustics 2012, Apr 2012, Nantes, France. hal-00810862

\section{HAL Id: hal-00810862 https://hal.science/hal-00810862}

Submitted on 23 Apr 2012

HAL is a multi-disciplinary open access archive for the deposit and dissemination of scientific research documents, whether they are published or not. The documents may come from teaching and research institutions in France or abroad, or from public or private research centers.
L'archive ouverte pluridisciplinaire HAL, est destinée au dépôt et à la diffusion de documents scientifiques de niveau recherche, publiés ou non, émanant des établissements d'enseignement et de recherche français ou étrangers, des laboratoires publics ou privés. 


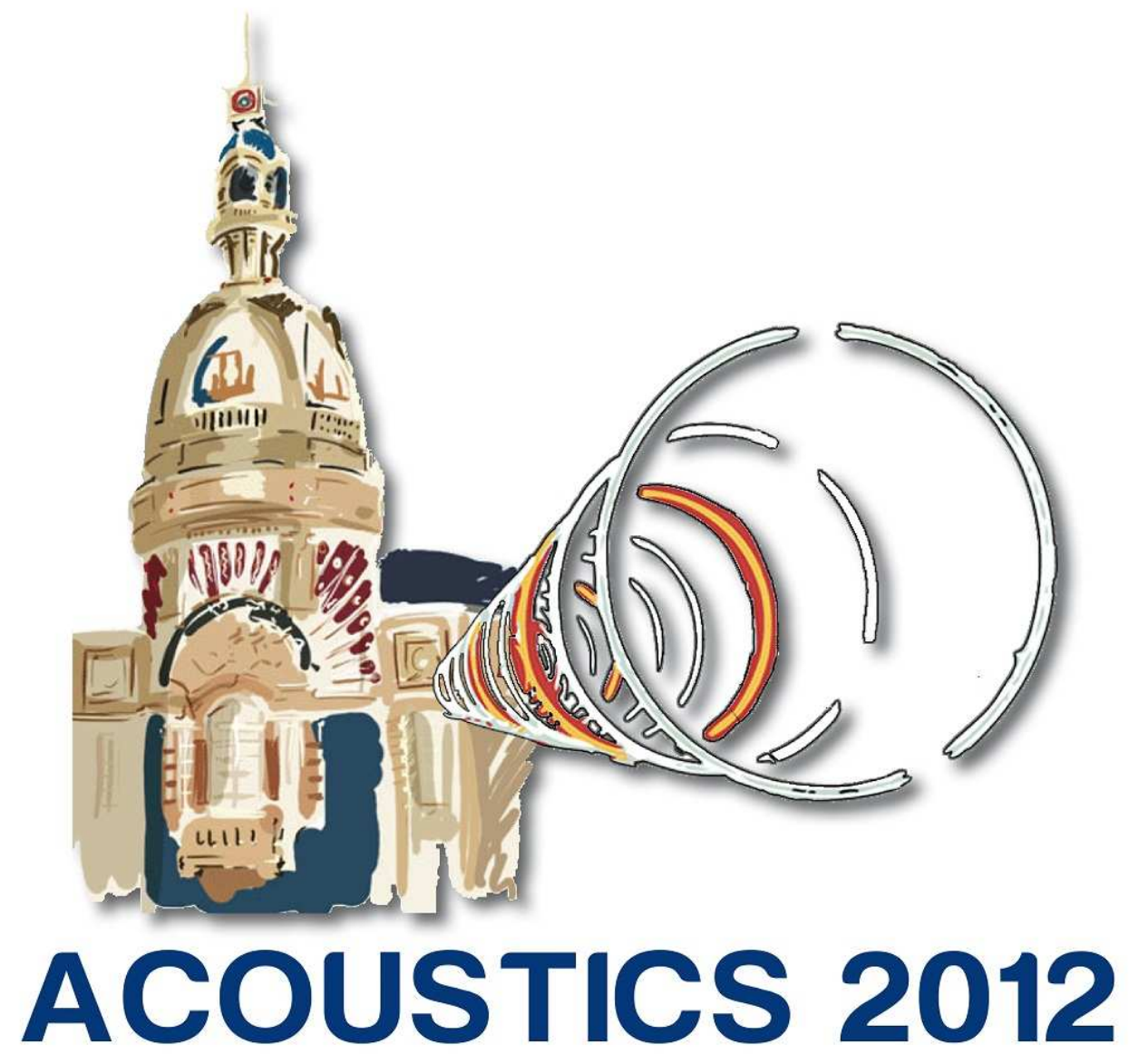

Acoustical inverse problems regularization: direct definition of filter factors using Signal-to-Noise Ratio

P. A. Gauthier, A. Gerard, C. Camier and A. Berry

GAUS, Université de Sherbrooke, 2500 Boul. de l'Université, Sherbrooke, Canada J1K2R1 philippe-aubert.gauthier@usherbrooke.ca 
Acoustic imaging aims at localization and characterization of sound sources using microphone arrays. In this paper a new regularization method for acoustic imaging by inverse approach is proposed. The method first relies on the singular value decomposition of the plant matrix and on the projection of the measured data on the corresponding singular vectors. In place of regularization using classical methods such as truncated singular value decomposition and Tikhonov regularization, the proposed method involves the direct definition of the filter factors on the basis of a thresholding operation, defined from the estimated measurement noise. The thresholding operation is achieved using modified filter functions. It has the advantage of simplifying the selection of the best regularization amount. Theoretical results show that this method is promising, in terms of ease of implementation and accuracy of results, in comparison with Tikhonov regularization and truncated singular value decomposition.

\section{Introduction}

Acoustic imaging aims at localization and characterization of sound sources using microphone arrays. Among the most common techniques for which commercial systems currently exists, one finds: beamforming [1,2], nearfield acoustical holography (NAH) [3] and inverse methods [4, 5, 6, 7, $8,9]$. One advantage of the inverse approach and conformal NAH for arbitrary geometries [3] is the possible use of non-uniform or source-conformal microphone arrays. Our attention is focused on 1) practical inverse problems which involve a finite number of sensors and 2) numerical inversion of matrix-form problems subject to measurement noise and random errors [6]. In this case, the direct system is described by a plant matrix $\mathbf{G} \in \mathbb{C}^{M \times L}$ ( $M$ microphones, $L$ sources $)$ that must be inverted. Major limitations and issues encountered in inverse methods in acoustics originate from the illconditioning of this matrix and lead to a large sensitivity of results to measurement noise. To circumvent this problem, solution regularization is introduced.

Although there are very few systematic ways to compare several regularization methods [10], comparison of the filter factors is often used to compare the behavior of various regularization methods. The filter factors are the coefficients (smaller than or equal to unity) that filter out the small singular values of $\mathbf{G}$ that may amplify measurement noise and random errors in the inverse solution. To the authors' knowledge most of the regularization schemes lead to filter factors that depend on the relative magnitudes of the singular values and on the regularization amount, not on the mesurement data and noise [10]. In this paper, we are concerned with a thresholding regularization that is directly related to data and signal-to-noise ratio in place of singular values.

The idea of working with a thresholding singular values, or coefficients, rejection is not new, but it was explored in a different way than the one presented in this paper. An example is found in the work of Thite and Thomson [7] who investigated the quantification of structure-borne transmission paths by inverse methods. They present the selection of a singular value rejection threshold based on either 1) the estimation of the error in the system identified frequency transfer functions (FRFs) or 2) the response error. The former approach can be used because the authors deal with a measured direct system (on the basis of identified FRFs) where an estimation of the error is possible. In this paper, it is assumed that the plant matrix is derived from a model. Hence, the former method does not apply. The second method presented by Thite and Thomson [9] is based on the idea that if a given singular value of $\mathbf{G}$ (plant matrix) contributes to the measured response less than the expected measurement noise, it should be discarded. This is formalized through a criterion that compares the estimated noise level with the actual signal level scaled by the first singular value of the plant matrix [9]. For the aforementioned methods, the singular value rejection operates from a given singular value order $i$ up to the system rank. In this paper, for the thresholding operation, a direct comparison between the estimated measurement noise level and the corresponding singular coefficients (projection of the measurement data on the singular vectors of $\mathbf{G}$ ) is performed for each singular value order $i$. In other words, a low order singular value coefficient dominated by noise can be discarded while a higher-order singular value coefficient with a significant signal-to-noise ratio can be conserved. The method also shares some conceptual backgrounds with subspace methods such as MUSIC [2] in the sense that we want to reject measurement noise, but the noise rejection is achieved using a different approach.

The presented method is also distinctive in terms of its underlying hypothesis. Indeed, as for NAH, many regularization methods typically a priori assume that measurement noise and random errors will dominate singular coefficients that involve more spatial variations (corresponding to small singular values or to large wavenumber (typically outside the radiation circle)). In the case of $\mathrm{NAH}$, this hypothesis is formalized through a low-pass k-space filter [3].

\section{The acoustical direct problem}

The direct discrete sound radiation problem is defined by

$$
\mathbf{p}=\mathbf{G s}_{\text {exact }},
$$

with $\mathbf{p} \in \mathbb{C}^{M \times 1}, \mathbf{s}_{\text {exact }} \in \mathbb{C}^{L \times 1}$ and $\mathbf{G} \in \mathbb{C}^{M \times L}$. In. Eq. (1), the exact source terms are $\mathbf{s}_{\text {exact }}$, the plant or sound radiation model is matrix $\mathbf{G}$ and the resulting sound pressure at the $M$ sensor locations are stored in vector $\mathbf{p}$. The number of sources in the model is noted $L$.

A typical configuration is shown in Fig. 1. It includes 49 microphones. The source model is made of 49 monopole sound sources. Microphones and sources are separated along $x_{1}$ and $x_{2}$ by $0.1 \mathrm{~m}$. Source array and sensor array are separated by $0.2 \mathrm{~m}$ along $x_{3}$. Both the source array and the sensor array are uniform square arrays. This configuration was studied by Nelson and Yoon [4] to evaluate the effect of various geometrical parameters on the conditioning of inverse problems. For the numerical results reported in this paper, the exact solution $\mathbf{s}_{\text {exact }}$ that we look for (on the basis of the 49-source model) involves the 12 active sources (with superimposed white circles in Fig. 1) with amplitudes set to unity.

The Singular Value Decomposition (SVD) of $\mathbf{G}$ is given by

$$
\mathbf{G}=\mathbf{U} \Sigma \mathbf{V}^{H}
$$

with $\mathbf{U} \in \mathbb{C}^{M \times M}, \mathbf{V} \in \mathbb{C}^{L \times L}$ and $\Sigma \in \mathbb{R}^{M \times L}$. Superscript ${ }^{H}$ denotes Hermitian transposition. Matrices $\mathbf{U}$ and $\mathbf{V}$ are unitary 


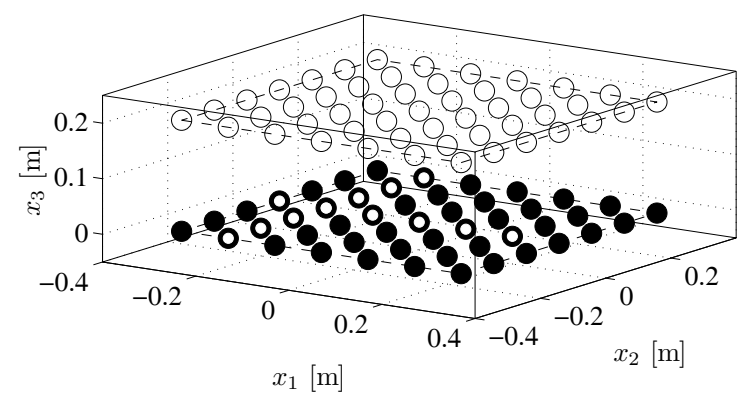

Figure 1: A typical free-field source and microphone configuration used for illustration purposes and for the numerical examples. The monopole sound sources are shown as black-filled circles and the microphones are shown as white-filled circles. Active sources in the exact solution

$\mathbf{S}_{\text {exact }}$ are indicated by superimposed white circles.

matrices and $\Sigma$ is a rectangular matrix with the real singular values $\sigma_{i}$ placed in decreasing order on its main diagonal. Using the SVD of $\mathbf{G}$, one writes Eq. (1) in matrix form

$$
\mathbf{p}=\sum_{i=1}^{\operatorname{rank}(\mathbf{G})} \mathbf{u}_{i} \sigma_{i} \mathbf{v}_{i}^{H} \mathbf{S}_{\text {exact }}
$$

where $\mathbf{v}_{i}^{H} \mathbf{S}_{\text {exact }}$ is the projection of the exact source solution on the orthonormal vector basis $\mathbf{v}_{i}$ and where $\operatorname{rank}(\cdot)$ is the matrix rank. The singular values $\sigma_{i}$ represent the coupling of the transformed source distributions $\mathbf{v}_{i}^{H} \mathbf{s}_{\text {exact }}$ with the transformed pressures $\mathbf{u}_{i}^{H} \mathbf{p}$ (indeed: $\mathbf{u}_{i}^{H} \mathbf{p}=\sigma_{i} \mathbf{v}_{i}^{H} \mathbf{S}_{\text {exact }}$ ).

\section{The acoustical inverse problem}

We now assume that $\mathbf{p}$ represents a sound field measured at $M$ locations. The measured sound field $\mathbf{p}$ also includes measurement noise. The objective of inverse sound radiation problems is to find a source distribution $\mathbf{s}$ that optimally predicts the measured sound field $\mathbf{p}$. This is formulated as follows

$$
\mathbf{p} \approx \hat{\mathbf{p}}=\mathbf{G s}
$$

where $\hat{\mathbf{p}}$ is the predicted sound pressure field at the $M$ points.

The inverse problem solution is generally obtained using the pseudo-inverse of the plant matrix $\mathbf{G}$

$$
\mathbf{S}=\mathbf{G}^{+} \mathbf{p}
$$

where ${ }^{+}$indicates pseudo-inversion [10]. Using the SVD of G, Eq. (5) is written

$$
\mathbf{s}=\sum_{i=1}^{\operatorname{rank}(\mathbf{G})} \frac{\mathbf{u}_{i}^{H} \mathbf{p}}{\sigma_{i}} \mathbf{v}_{i} .
$$

Most inverse problems are ill-posed [4, 5, 10]: the singular values typically span many orders of magnitude and the very small singular values may over-amplify the corresponding contribution of $\left(\mathbf{u}_{i}^{H} \mathbf{p}\right) \mathbf{v}_{i}$. There is therefore a need for solution regularization [10]. Any regularization strategy (including classical Tikhonov regularization, Tikhonov regularization with discrete smoothing norm, truncated singular value decomposition [10]) will lead to a modified version of Eq. (6) in the form

$$
\mathbf{s}=\sum_{i=1}^{\operatorname{rank}(\mathbf{G})} f_{i} \frac{\mathbf{u}_{i}^{H} \mathbf{p}}{\sigma_{i}} \mathbf{v}_{i},
$$

where the $f_{i}$ are the filter factors [10]. The filter factors range from zero to unity. Filter factors smaller than one artificially damp the corresponding singular components $\left(\mathbf{u}_{i}^{H} \mathbf{p} / \sigma_{i}\right) \mathbf{v}_{i}$ in the solution $\mathbf{s}$.

\subsection{Truncated singular value decomposition}

One of the most basic regularizations involves the truncation of the SVD of $\mathbf{G}$. Then, the filter factors are [10]

$$
f_{i}=\left\{\begin{array}{l}
1 \forall i \leq K \\
0 \forall i>K,
\end{array}\right.
$$

where $K$ is the truncation order which operates as a regularization parameter. This parameter selection will also typically depend on the plant matrix, the singular values and the measurement noise. Finding the best truncation order $K$ is not easy and specific methods are designed for that specific purpose $[7,9]$. Typical truncated singular value decomposition (TSVD) filter factor shapes are shown in Fig. 2(a) and (b). In this type of regularization, filter factors abruptly pass from unity to zero at $i=K+1$. Therefore TSVD can be interpreted as a regularization method that uses Heaviside step functions as filter factors for which singular values smaller than $\sigma_{K}$ are cancelled. Regularization based on TSVD is mostly suited for rank-deficient problems where the numerical rank [11] is lower than the smallest dimension of $\mathbf{G}$.

\subsection{Classical Tikhonov regularisation}

The classical Tikhonov regularization with regularization parameter $\lambda$ gives the following filter factors [10]

$$
f_{i}=\frac{\sigma_{i}^{2}}{\sigma_{i}^{2}+\lambda^{2}}=\frac{1}{1+\lambda^{2} / \sigma_{i}^{2}}
$$

These filter factors are a direct consequence of the standard formulation of the inverse problem with Tikhonov regularization in the form of a quadratic minimization problem

$$
\mathbf{s}=\operatorname{argmin}\left\{\|\mathbf{p}-\hat{\mathbf{p}}\|_{2}^{2}+\lambda^{2}\|\mathbf{s}\|_{2}^{2}\right\}
$$

where $\|\mathbf{p}-\hat{\mathbf{p}}\|_{2}^{2}$ involves a minimization of the prediction errors, in the least-mean-square sense, at the sensor array and $\lambda^{2}\|\mathbf{S}\|_{2}^{2}$ penalizes the solution 2-norm. The regularization parameter $\lambda$ controls the amount of regularization. Further details can be found in [10].

Typical filter factors of Tikhonov regularization are shown in Fig. 2(c) and (d). These generic shapes are much smoother than the generic TSVD step-like filter factors. Also, they have the advantage of automatically imposing a threshold on the acceptable singular values. Indeed, any singular coefficient $\mathbf{u}_{i}^{H} \mathbf{p}$ that is associated with a singular value for which $\sigma_{i} \leq \lambda$ will be filtered out by the filter factors. For very small singular values, $f_{i} \approx \sigma_{i}^{2} / \lambda^{2}$. According to the last expression in Eq. (9), the filtering operation solely depends on the penalization-to-singular-value ratio $\lambda / \sigma_{i}$. This confirms the observation in the introduction, that Tikhonov regularization is directly related to the singular values. In the next section, a similar function for the filter factor will be introduced, but this time in terms of signal-to-noise ratio. Tikhonov regularization is often used for general ill-posed problems where the condition number $\kappa(\mathbf{G})=\sigma_{\max } / \sigma_{\min }$ is large $\left(\sigma_{\max }\right.$ and $\sigma_{\min }$ being the largest and the smallest singular values, respectively). 

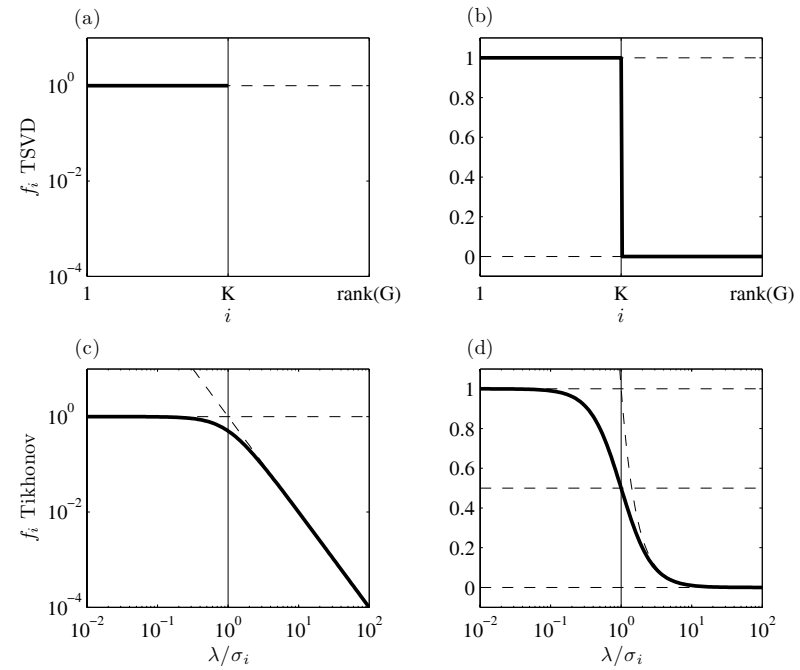

Figure 2: Generic representations of filter factors. Left: Logarithmic vertical scale. Right: Linear vertical scale. (a) and (b): TSVD filter factors for a truncation order $K$. (c) and (d): filter factors obtained for Tikhonov regularization

in terms of normalized regularization parameter $\lambda / \sigma_{i}$.

\section{Direct definition of the filter factors using signal-to-noise ratio}

The behavior of the filter factors as defined above is a direct consequence of the selected regularization strategy. In direct regularization methods such as TSVD and Tikhonov regularization, the selection of the best regularization parameter $K$ or $\lambda$ is not a trivial task [5, 7, 9]: it depends on both the regularization strategy and the measured data. Several regularization parameter selection methods in acoustics have been investigated by Nelson and Yoon [4]. Most common direct regularization strategies involve a comparison of the regularization parameter with the singular values, and not the measured sound field. In all regularization strategies, the main objective is to cancel in Eq. (7) the contribution of the singular components that are dominated by measurement noise. Since the coefficients $\mathbf{u}_{i}^{H} \mathbf{p}$ stabilize to a value equal to the measurement noise effective value $\sigma_{\text {noise }}[10]$, it would be interesting to artificially define a set of filter factors that would automatically discard the coefficients $\mathbf{u}_{i}^{H} \mathbf{p}$ lower than or in the range of $\sigma_{\text {noise }}$. This is the purpose of the presented method. Hence, for the proposed method, $\mathbf{G}, \mathbf{p}$ and $\sigma_{\text {noise }}$ must be known in order to implement this regularization. Note that this thresholding regularization method is different to the ones reported in [7,9] since in these references the authors seek for a rejection threshold that would give the best TSVD order $K$.

Also note that this inverse problem regularization method has similarities with previous regularization techniques. Indeed, in the literature on general inverse problem, one finds many regularization strategies that are not defined through typical quadratic optimization problems (as for Eq. (10)) but are derived from target performances for various type of application [10]. Assuming that the measurement noise effective value $\sigma_{\text {noise }}$ is known, either from direct measurement or from an estimation, it is possible to proceed to the next step.

In Sec. 3.2, it was noted that the classical Tikhonov filter factors have a simple definition (Eq. (9)) and depend on the ratio $\lambda / \sigma_{i}$. In this section, we keep a similar structure for the filter factors, but we use a new ratio $R$. We then write

$$
f_{i}=\frac{1}{1+R} \text {. }
$$

As for the filter factors shown in Fig. 2(c), this function goes from one to zero as $R$ goes from 0 to $\infty$. It is desirable to relate this ratio $R$ to the signal-to-noise ratio for each of the individual singular coefficients $\mathbf{u}_{i}^{H} \mathbf{p}$. Accordingly, the filter factors of Eq. (11) can be written as follows

$$
f_{i}\left(\sigma_{\text {noise }}\right)=\frac{1}{1+R_{i}^{-\alpha}}
$$

with the signal-to-noise ratio $R_{i}=\mathbf{p}^{H} \mathbf{u}_{i} \mathbf{u}_{i}^{H} \mathbf{p} / \sigma_{\text {noise }}^{2}$ and $\alpha \geq$ 0 . The transition rate (toward one or zero) is controlled by the scaling coefficient $\alpha$ in Eq. (12). In Eq. (12), when the coefficients $\mathbf{u}_{i}^{H} \mathbf{p}$ are large compared to the noise effective value $\sigma_{\text {noise }}$, the $R_{i}^{-\alpha}$ are small and the filter factors converge towards one. When the coefficients $\mathbf{u}_{i}^{H} \mathbf{p}$ are small compared to the noise level, the $R_{i}^{-\alpha}$ are large and the filter factors converge towards zero. Moreover, the threshold from which the coefficients are filtered is automatically defined by the estimated noise level which greatly simplifies the regularization parameter selection.

However, it is known that the coefficients $\mathbf{u}_{i}^{H} \mathbf{p}$ tend to stabilize to the noise effective value as $i$ increases [10]. Therefore, according to Eq. (12), the imposed filter factors $f_{i}\left(\sigma_{\text {noise }}\right)$ would simply tend to the value $1 / 2$. Such a value does not eliminate the singular components that are subject to measurement noise in Eq. (7). This is exemplified in Fig. 3 for the source and microphone configuration shown in Fig. 1 at $800 \mathrm{~Hz}$. In this example, the exact solution involves a set of active sources (Fig. 1) with unit monopole amplitude. Figure 3 shows the coefficients $\mathbf{u}_{i}^{H} \mathbf{p}$ as function of $i$ for a case without noise and with Gaussian noise with a standard deviation equal to 0.005 . These coefficients tend to globally decrease with $i$. In the case with measurement noise, the $\mathbf{u}_{i}^{H} \mathbf{p}$ asymptotically tend to the noise level of $0.005 \mathrm{~Pa}$. Also, in the case without noise, some of these coefficients are very small (outside the figure range). This is a direct consequence of the active source configuration that creates a sound field which is potentially orthogonal to some of the singular vectors $\mathbf{u}_{i}$. We now proceed with modified filter factors based on the signal-to-noise ratio with an additional offset coefficient that will tackle the above limitation.

Since the $f_{i}$ defined in Eq. (11) vary from one to zero for $R$ varying between roughly $R=10^{-3}$ to $R=10^{3}$, the scaling coefficient is set to $\alpha=4$ and a supplementary offset $\beta=0.75$ is introduced. The following filter factor is proposed

$$
f_{i}\left(\sigma_{\text {noise }}\right)=\frac{1}{1+\left(10^{-\beta} R_{i}\right)^{-\alpha}} .
$$

In this case, the ratios $R_{i}=\mathbf{p}^{H} \mathbf{u}_{i} \mathbf{u}_{i}^{H} \mathbf{p} / \sigma_{\text {noise }}^{2}$ that are at least $10^{\beta}$ are left unfiltered (corresponding to $f_{i} \geq 0.5$ ) while the ratios $R_{i}$ that are equal or lower than $10^{\beta}$ are filtered $\left(f_{i} \leq\right.$ $0.5)$. With $\beta=0.75$, this threshold corresponds approximately to $5\left(10^{0.75}=5.6234\right)$. The selection of $\alpha$ controls the transition rate from the passband to the stopband and the offset $\beta$ adjusts the threshold from which the filter factors will pass from smaller than to larger than $1 / 2$. When $\alpha$ becomes very large, the filter factors Eq. (13) will tend to a Heaviside step function. These simple features highlight the interest of introducing the signal-to-noise ratio in place of the $\lambda / \sigma_{i}$ ratio in filter factors. 


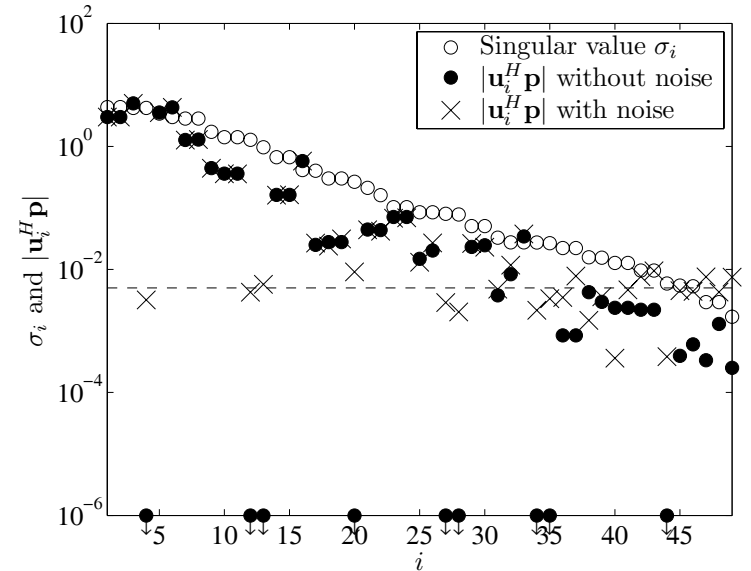

Figure 3: Singular values $\sigma_{i}$ and singular coefficients $\mathbf{u}_{i}^{H} \mathbf{p}$ $[\mathrm{Pa}]$ without noise and with noise $\left(\sigma_{\text {noise }}=0.005 \mathrm{~Pa}\right)$.

Sound pressure $\mathbf{p}$ is created using Eq. (1) for the configuration shown in Fig. 1 at $800 \mathrm{~Hz}$. Coefficients $\mathbf{u}_{i}^{H} \mathbf{p}$ with measurement noise tends to $0.005 \mathrm{~Pa}$ (dashed line) which corresponds to $\sigma_{\text {noise }}$.

The filter factors as defined above using the SNR ratio and Tikhonov regularization are shown in Fig. 4 for the example mentioned above with measurement noise. In the case of Tikhonov regularization, the regularization parameter $\lambda$ was set equal to $\sigma_{30}$ so that the Tikhonov regularization effectively filters out the components $\mathbf{u}_{i}^{H} \mathbf{p}$ that are dominated by noise from $i=31$ to $i=49$ (see Fig. 3). On Fig. 4, one notes that the filter factors obtained from the signal-to-noise ratio and Eq. (13) will effectively filter out the components that are in the range of the noise level. For the coefficients $\mathbf{u}_{i}^{H} \mathbf{p}$ that are in the range of the noise level (shown as dashed lines in Figs. 3 and 4), the SNR approach filters out much more strongly than the Tikhonov regularization. Also, one interest and distinction of the filter factors based on the SNR is that they also filter noisy components even if they are associated with large singular values. An example of this property is clearly visible in Fig. 4 for $i=4$. This type of behavior cannot arise in singular value rejection methods such as those proposed in [7, 9]. From this behavior, we expect to obtain less spatial low-frequency noise in the solution with the SNR filter factors. Moreover, higher-order coefficients (in the range of $i=30$ ) with significant SNRs are preserved with the proposed method. This is not the case with the Tikhonov or TSVD regularization. Therefore, we also expect a sharper solution with the proposed method.

\section{Numerical examples}

In this section, we compare the inverse problem solutions s obtained from pseudo-inversion, TSVD, classical Tikhonov regularization and the SNR filter factors for the basic source and receiver configuration presented by [4] and shown in Fig. 1. The direct and inverse problems involve 49 unknown sources and the exact solution involves only 12 active sources. The assumed sound pressure was evaluated using Eq. (1) and measurement noise was added to $\mathbf{p}$ as a zero-mean Gaussian random process with standard deviation $\sigma_{\text {noise }}$. The goal is to retrieve the 12 active sources from the 49 candidates in the inverse problem.

The mapping of the exact and inverse solutions in the

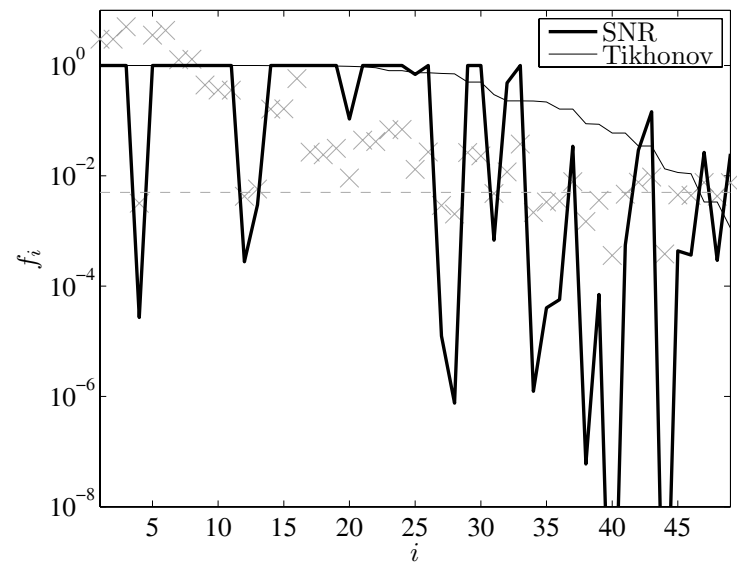

Figure 4: Filter factors obtained using SNR (Eq. (13)) and Tikhonov regularization (Eq. (9)) for the numerical example reported in Fig. 1. Grey lines and markers are copies of the coefficients $\mathbf{u}_{i}^{H} \mathbf{p}$ with noise (Fig. 3).

source plane is shown in Fig. 5. Clearly, the TSVD, Tikhonov and filter factors based on SNR solutions all approach the exact solution to some extent. As expected, the pseudo-inverse solution (Eq. (5)) provides an incorrect estimation. The proposed method with the SNR filter factors is effective and provides a more accurate description of the sources than the other methods.

To quantify the accuracy of the inverse solutions, the relative error norm $\left\|\mathbf{s}_{\text {exact }}-\mathbf{s}\right\|_{2} /\left\|\mathbf{s}_{\text {exact }}\right\|_{2}$ is introduced in Tab. 1 . From this performance metric, we see that the most efficient method is the filter factors based on SNR. It is worth noting that this method outperforms the two most common regularization methods: TSVD and Tikhonov regularization, for source imaging.

To illustrate how the method effectively approximates the measured sound pressure $\mathbf{p}$ at the microphone array, the relative residual norm $\|\mathbf{p}-\hat{\mathbf{p}}\|_{2} /\|\mathbf{p}\|_{2}$ on the predicted sound pressure is reported in Tab. 1. Clearly, the pseudo-inverse solution very well approximates the initial acoustic pressure. This is expected since the pseudo-inverse solution should provide $\hat{\mathbf{p}}=\mathbf{p}$ ( since $M=L$ ) at the cost of a nearly instable inverse problem solution $\mathbf{s}$. The two classical methods, namely the TSVD and Tikhonov regularization lead to the largest prediction errors. This was also expected since these methods involve a progressive filtering ( $f_{i}$ gets smaller as $i$ increases from $\sigma_{i}=\lambda$ ) of the measured data to stabilize the solution. The method based on SNR also outperforms the others in terms of prediction error at the microphone array.

\section{Conclusion}

In this paper, a new regularization method for acoustical inverse problem was proposed and illustrated in a simple source identification problem. The proposed method is not primarily meant to increase spatial resolution or accuracy of the solution but to provide a regularization that is directly derived from a comparison of the singular coefficients with the measured or estimated noise level. The proposed method relies on the direct definition of the filter factors with a threshold value related to the estimated noise level. It was shown that the proposed method performs at least as effectively as the TSVD or Tikhonov methods. 
(a)

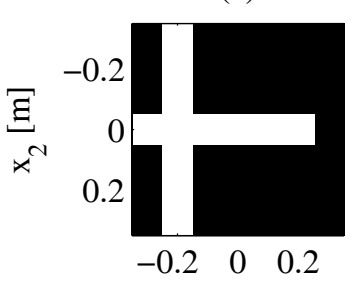

(b)

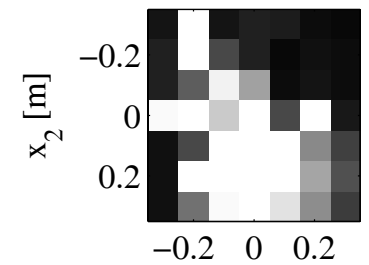

(d)

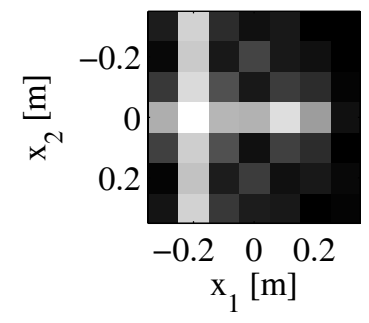

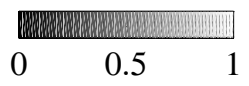

(c)

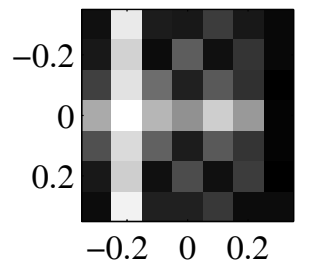

(e)

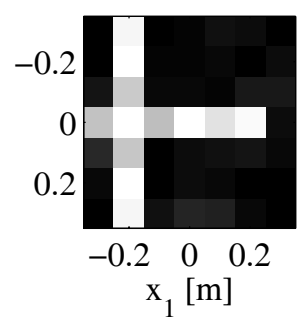

Figure 5: Magnitudes of exact solution $\mathbf{S}_{\text {exact }}$ (monopole amplitude $[\mathrm{Pa} \mathrm{m}]$ ) and various solutions $\mathbf{s}$ (monopole amplitude $[\mathrm{Pa} \mathrm{m}]$ ) with $\sigma_{\text {noise }}=0.005 \mathrm{~Pa}$. (a): exact solution; (b): pseudo-inverse solution (Eq. (5)); (c) TSVD solution with $K=30$; (d): Tikhonov solution and with

$$
\lambda=0.0506=\sigma_{30} \text {, and (e): SNR } f_{i} \text { solution. }
$$

To achieve a more efficient regularization, it could be interesting to combine the proposed method with other approaches such as singular value rejection methods like those proposed by Thite and Thompson [7]. It could also be possible to combine the proposed method with the scaling method presented by Moorhouse [6]. In this scaling method, it is assumed that the most significant measure is the mean sound pressure at the microphone array. The resulting inverse problem solution is then scaled to ensure that the reconstructed mean sound pressure is equal to the measured mean sound pressure.

The proposed method could also be extended to acoustic imaging methods, such as conformal NAH or HELMS (Helmholtz Least-Mean-Square) where matrix inversion is also performed. This is a topic of curent developments.

Since the proposed method mostly focused on measurement noise, further work could be done to evaluate the effect of other types of random errors on plant matrix $\mathbf{G}$ (microphone positioning, phase mismatch, etc.) and to possibly define a new thresholding regularization method that would fit the case of random errors in microphone array measurements. Systematic study of the parameters $\alpha$ and $\beta$ should also be done.

Current work, not reported in this paper, studies the efficiency of the proposed method with varying noise level. This is the topic of a current manuscript. Preliminary results are promising.

Table 1: Performance of the regularization methods based on the relative error norms at the source distribution $\left\|\mathbf{s}_{\text {exact }}-\mathbf{s}\right\|_{2} /\left\|\mathbf{s}_{\text {exact }}\right\|_{2}$ and on the relative residual norms at the microphone array $\|\mathbf{p}-\hat{\mathbf{p}}\|_{2} /\|\mathbf{p}\|_{2}$.

\begin{tabular}{|l|l|l|}
\hline Regularization & $\begin{array}{l}\text { Relative error } \\
\text { norm }\end{array}$ & $\begin{array}{l}\text { Relative } \\
\text { residual norm }\end{array}$ \\
\hline $\begin{array}{l}\text { Pseudo- } \\
\text { inverse }\end{array}$ & 1.6611 & $3.961 \times 10^{-14}$ \\
\hline TSVD & 0.43171 & 0.005088 \\
\hline Tikhonov & 0.368 & 0.0052601 \\
\hline SNR & 0.21916 & 0.002824 \\
\hline
\end{tabular}

\section{References}

[1] B.D. Van Veen, K.M. Buckley, "Beamforming: A versatile approach to spatial filtering", IEEE ASSP Mag 5(2) 4-24 (1988).

[2] H. Teutsch, Modal Array Signal Processing: Principles and Application of Wavefield Decomposition, Springer, Berlin (2007).

[3] E.G. Williams, Fourier Acoustics - Sound Radiation and Nearfield Acoustical Holography, Academic Press, San Diego (1999).

[4] P.A. Nelson, S.H. Yoon, "Estimation of acoustics source strength by inverse method: Part I, conditioning of the inverse problem", J. Sound Vib. 233(4), 643-668 (2000).

[5] S.H. Yoon, P.A. Nelson, "Estimation of acoustics source strength by inverse method: Part II, experimental investigation of methods for choosing regularization parameters", J. Sound Vib. 233(4), 669-705 (2000).

[6] A.T. Moorhouse, "Compensation for discarded singular values in vibro-acoustic inverse methods", J. Sound Vib. 267(2) 245-252 (2003).

[7] A.N. Thite, D.J. Thompson, "The quantification of structure-borne transmission paths by inverse methods. Part 1: Improved Singular value rejection methods", $J$. Sound Vib. 264(2) 411-431 (2003).

[8] A.N. Thite, D.J. Thompson, "The quantification of structure-borne transmission paths by inverse methods. Part 2: Use of regularization techniques", J. Sound Vib. 264(2) 433-451 (2003).

[9] M.H.A. Janssens, J.W. Verheij, T. Loyau, ’Experimental examples of pseudo-forces method used in chracterisation of a structure-borne sound source", Appl. Acoust. 63(1) 9-34 (2002).

[10] C. Hansen, Rank-Deficient and Discrete Ill-Posed Problems, SIAM, Philadelphia (1998).

[11] G.H. Golub, C.F. van Loan, Matrix Computations, John Hopkins University Press, Baltimore (1996). 